\title{
中国心理学前沿
}

\section{心理应激反应新指标：线粒体 DNA}

\author{
陆 梭 ${ }^{1,2^{*}}$ 刘正奎 ${ }^{1}$ \\ 1. 中国科学院心理研究所, 北京; \\ 2. 中国科学院大学心理系, 北京
}

邮箱: lus@psych.ac.cn

摘 要: 心理应激是造成多种心身疾病的重要因素, 有效应对心理应激是心理 健康的关键。现有研究表明线粒体是对心理应激反应最为敏感的细胞器。应激 暴露会导致每个细胞的线粒体含量和功能的变化。心理应激主要通过激活 HPA 轴以及神经分泌因素等方面进行相互调节。本文旨在说明在心理应激尚未导致 线粒体功能严重损伤和机体内稳态失调之前, 研究者就应及时关注线粒体 DNA 含量及其功能指标变化的意义。从而进一步探讨把改善线粒体功能作为靶向目 标的心理应激千预策略的可能性, 优化当前的心理应激领域研究。

关键词: 心理应激; 线粒体 DNA 掿贝数; 线粒体功能; 应激反应; 生理指标 投稿日期：2019-05-28；录用日期：2019-06-21；发表日期：2019-09-10 


\title{
A new Index of Psychological Stress Reaction: Mitochondrial DNA
}

\author{
Lu Suo $^{1,2 *}$ Liu Zhengkui ${ }^{1}$
}

1. Institute of Psychology, Chinese Academy of Science, Beijing;

2. Department of Psychology, University of Chinese Academy of Science, Beijing

Abstract: Psychological stress is an important factor causing a variety of psychosomatic diseases. Effective coping with psychological stress is the key to mental health. Existing studies show that mitochondria are the most sensitive organelles for psychological stress response. Psychological stress may leading to changes either in mitochondrial content per cell, or in mitochondrial functional capacity. Psychological stress mainly regulates each other by activating the HPA axis and neurosecretory factors. The purpose of this article is to show that before psychological stress has not caused serious damage of mitochondrial function and the imbalance of homeostatic, the scientific research personnel should be paid to the significance of changes in mitochondrial DNA copy number and its functional indicators. Therefore, the possibility of improving the mitochondria function as a targeted psychological stress intervention strategy is further explored to optimize the current research in the field of psychological stress.

Key words: Psychological stress; Mitochondrial DNA copy number; Mitochondrial function; Stress response; Physiological index

Received: 2019-05-28; Accepted: 2019-06-21; Published: 2019-09-10 
Copyright $\odot 2019$ by author(s) and SciScan Publishing Limited.

This work is licensed under a Creative Commons Attribution 4.0 International License. https://creativecommons.org/licenses/by/4.0/

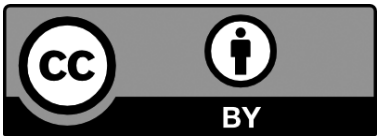

\section{1 引言}

线粒体是一种存在于真核生物细胞质中，含有核外遗传物质的半自主性细 胞器 [1]。作为细胞的动力工厂, 超过 $90 \%$ 的细胞能量都由线粒体产生, 它可 通过能量转化和细胞内信号传导来维持生命正常运行。近年来，线粒体含量及 其功能指标已经成为职业与环境暴露 [2]［3］［4]、应激 [5]［6]［7] 等研究领域的重要组成部分。相对于反映特定生物过程的传统生物标记物而言, 线粒体的功能复杂多样, 它是除了细胞核以外唯一含有 DNA 的细胞器, 主要负 责提供生物体内有关生物合成、呼吸、分泌及机械运动等全部细胞活动所需要 的化学能 [8]。近十年来的研究表明, 由遗传和生化作用影响所导致的线粒体 缺陷问题，人体多个系统会因此出现各种临床病症 [9]，通过大量研究已证实， 线粒体 DNA 拷贝数 ( mitochondrial DNA copy number, mtDNAcn) 及其功能变化 能够直接影响机体内部系统的代谢调节 [10 ]、能量转化 [11]、脑功能 [12]、 免疫激活 $[13 ］ ［ 14 ＼mathrm{~ 、 细 胞 癌 变 ~}[15 ］ ［ 16] ［ 17 ］ ［ 18 ］$ 以及衰老速率和寿 命 $[19][20][21][22]$ 。因此, 线粒体 DNA 含量及其功能的变化可作为 多种病症的生物标记物。

应激是个体内部稳态受到威胁时的一种状态，表现为个体产生一系列适应 性补偿反应来维持机体内部稳态 [23]。然而，持续的心理应激状态可能会对 大脑功能和人类行为带来严重和持久的不利影响 [24] [25]。应激领域研究, 首先, 从测量学角度考虑主要有三种对应激的测量方式：规定情境定义法、生 理指标检测法和心理量表评定法 [26]。其次，从应激病理生理学的角度来看， 研究主要围绕大脑、内分泌和免疫系统三个方向进行 $[27]$ 。最后, 从神经心 
理学角度出发的研究已经证实, 个体的生活经历以及应激暴露经验会导致亚细 胞发生多种变化，包括端粒形态改变 ［28］、免疫激活或抑制［29］［30］、 调控基因表达 [31］、致癌特性［32］［33］和表观遗传过程［34］［35］。由 人类为被试的实验研究表明，严重的心理应激会对线粒体结构和功能造成不利 影响, 并与 mtDNAcn 和功能变化有关 [36 ] [37 ]。因而, 及时关注 mtDNAcn 及功能水平的变化, 对心理应激研究领域将带来有益推动。

已有大量研究表明运动训练既是与年龄相关的线粒体功能障碍的预测因素, 也可能是预防或减缓由衰老引发的线粒体功能衰退的重要因素。现有的普遍共 识是老年人保持中等强度的耐力运动训练，能够改善包括 mtDNAcn、线粒体酶 的活性等在内的线粒体含量和功能 [38 ]。虽然运动训练不能完全恢复与年龄 相关的线粒体功能 [39]，但能够减缓线粒体功能衰退的速率。因此在排除衰 老因素后，可以探索通过简单、有效的运动训练作为一种心理应激干预方法的 可能性，以恢复线粒体 DNA 含量及其功能。

线粒体在细胞稳态中起至关重要的作用，而在应激领域研究中要关注其作 为各种应激刺激首要反应物的地位, 突出它在急性和慢性心理应激反应中的作 用。线粒体作为心理应激诱导分子、细胞层面发生改变的前兆，特别是慢性心 理应激会导致线粒体功能损伤，进而诱发多种心身疾病。由于生物体内的不同 器官组织有着不同的能量代谢需求，故它们各自含有不同类型和功能的线粒体。 此外, 应激暴露可能独立地影响 mtDNAcn 和功能, 而线粒体功能变化也独立于 mtDNAcn 变化，这可能导致线粒体对不同应激类型的反应呈多样性，从而给细 胞和机体健康产生不同影响。因此，在心理应激尚未造成线粒体功能损伤和机 体内稳态失调之前，就应密切关注线粒体 DNA 含量及其功能水平变化，优化当 前心理应激领域研究。

\section{2 线粒体的结构和功能}

线粒体一般呈杆状或粒状，它由内外两层彼此平行且高度特化的单位膜包 围而成，膜中包围着线粒体基质。人类 mtDNA 含有 16,569 个核苷酸碱基对， 其中有 37 个基因分别编码 13 种蛋白质多肽、 2 种 rRNA 和 22 个 tRNA [ 40 ]。 
mtDNA 没有组蛋白保护，并缺乏有效的损伤修复功能，因此更容易受到外界刺 激因素的影响。线粒体基因组个数指的就是 mtDNAcn, 而每个线粒体当中一般 包含 2 10个 mtDNAcn [41］［42］。MtDNA 是编码呼吸链产生能量所必需的 基因。呼吸链中含有五种多蛋白酶复合物 I, II, III, IV 和 V, 而大多数线粒体 蛋白是在 mtDNA 中编码。整个呼吸链过程中释放的能量建立了维持生命的线 粒体电化学梯度（ $\Delta \Psi)$ ，而这就是生物必须进食和呼吸的根本原因。稳定的 mtDNAcn 对于维持正常生理状态很重要, 也能够对线粒体功能的损伤起缓冲作 用 $[43]$ 。

在应激作用下，线粒体膜形态会发生膨大。线粒体基质含有三羧酸循环和 $\beta$ - 氧化途径的酶, 它们主要功能是代谢包括脂肪和糖在内的摄人性能量。在人 类和动物中, mtDNA 是从母亲那里遗传的 [44]并且携带与种族、民族相关的 单核苷酸呈现多态性 [45]。MtDNA 没有松散的末端，因此不含有端粒 [46 ]。 由于 mtDNA 缺乏内含子，与核基因组相比它更容易受到损伤 [47]，这或许可 以解释它为什么容易受到应激的影响。线粒体的能量代谢功能主要通过消耗氧 来进行电子传递链（呼吸链），这说明从营养物质到质子泵 $(\mathrm{H}+)^{2}$ 的能量流动 过程中会产生电化学梯度，该过程一方面用于合成腺嘌呤核苷三磷酸（ATP）, 另一方面会生成活性氧（ROS）自由基对线粒体产生损害作用 [48］。

线粒体内膜上的呼吸链传递过程中所释放的能量是驱动多种不同功能的能 量来源。其中最重要的是 ATP 的产生 [49], 该过程可以促进大多数细胞的反应, 包括基因表达，离子动态平衡，蛋白质与激素合成、分泌，神经递质释放和再 摄取等方面。在应激条件下可通过腺苷酸活化蛋白激酶（AMPK）信号通路来调 控线粒体 ATP 的合成 $[50]$ 。线粒体在呼吸链复合物水平上使用氧的能力, 称 为线粒体氧化能力。因此, 线粒体氧化能力取决于呼吸链功能和 mtDNA 的完整。 ROS 在启动和调节细胞调亡的过程中发挥关键作用，而线粒体中氧自由基水平 就是呼吸链底物端和氧端电子漏动态平衡的结果。由此可见, 线粒体在能量代 谢中起着不可或缺的关键作用，其中 mtDNAcn 的变化可作为氧化应激水平的反 应指标。

线粒体除了具有能量代谢的作用外，它还具有维持生命的多种基本功能, 
从而影响细胞核内基因表达和机体生理调节。线粒体作为细胞内 ROS 的主要生 成者，它能够在较低水平上维持生命，但当它压制住抗氧化防御机制后，就可 能导致氧化应激反应，进而在神经退行性过程 [51] 和应激病理生理学中扮演 重要的角色。

\section{3 线粒体 DNA 对心理应激反应系统的调节}

在应激作用下，神经内分泌反应是进行能量代谢和多种器官功能变化的基 础。其中下丘脑 - 垂体 - 肾上腺皮质 ( HPA ) 轴和交感神经 - 肾上腺髓质 ( SAM ) 轴是最重要的神经内分泌反应激活系统。HPA 轴、SAM 轴都与大脑相连，应激 反应所需的多种激素是由它们产生 [52]。通过小鼠的研究表明，mtDNA 遗传 变异可能导致应激反应性激素皮质酮的产生数量发生显著变化 [53][54]。此外， 有研究者从具有线粒体氧化应激遗传突变的个体身上发现，他们集中表现出肾 上腺皮质功能障碍和皮质醇过低症状 [55]。由于遗传缺陷所导致线粒体 ATP 转运受损的患者，他们在静息状态下儿茶酚胺水平是健康对照组的两倍 [56 ]。 上述这些临床研究皆可说明, HPA 轴和 SAM 轴的具体活动可以通过线粒体功能 进行直接调节。线粒体的影响涵盖了心理应激反应的多个方面，其中包括糖皮 质激素 $[57$ ，促炎细胞因子 $[58$ ] 和儿茶酚胺 [59 ，而这些方面都与 HPA 轴、 SAM 轴密切相关，因此在应激研究领域，研究者应逐渐把线粒体视作应激反应 的一个客观、有效的生理指标。

同样，就神经内分泌反应的原因而言，部分是由交感神经和副交感神经两 个系统在多重组合条件下进行驱动。有关研究表明，在运动过程中 mtDNA 病症 患者的神经内分泌水平发生显著变化 $[60]$ 。这其中的原因可能是因为静息状 态下和运动期间迷走神经活动减少，而此方面的证据主要来自于在静息状态时 心率增加和线粒体疾病患者的高频率 R-R 间期变异性降低 [61 ]。在运动训练 期间，有 mtDNA 病症的患者也同时具有由 SAM 轴释放的应激反应性肾上腺素 和去甲肾上腺素显著升高的临床表现 $[62]$ ，这可能是因为线粒体缺陷改变了 个体对各类应激源在生理、心理方面的有效感知。

这些 mtDNA 异常的神经内分泌对运动的反应是否也能够转化、引申为心理 
应激的异常反应仍不清楚。然而, 通过健康个体的研究证据显示, 那些由躯体 活动表现出更强的 HPA 轴反应的个体，在心理应激时也表现出皮质醇释放量显 著增加 [63], 这就说明同一种生物学机制可以调节个体对不同性质的应激源 ( 身 体和心理）的反应程度。再者，不同的种族、民族群体之间，在心理应激反应 时的神经内分泌方面也存在显著的个体差异 [64]。值得关注的是 mtDNA 会因 种族、民族的不同而导致其存在差异 [45]，线粒体功能的差异也可以部分地 解释不同个体在心理应激反应时存在的差异。总而言之, 未来需要更多的研究 来深人探讨线粒体 DNA 含量和功能水平的差异与 HPA 轴、SAM 轴和自主神经 系统调节人类心理应激反应时产生个体间差异之间存在的关系。

\section{4 线粒体 DNA 含量和功能可作为评估心理应激 的客观指标}

\section{1 心理应激与线粒体功能的关系}

不论是急性还是慢性心理应激，都会引起线粒体含量和功能的变化。国外 研究者对 23 项有关线粒体功能障碍的研究进行元分析后表明, 严重或慢性心 理应激对线粒体形态、结构完整性有不利影响 [65]。通过小鼠研究发现，急 性心理应激会导致肠道线粒体功能损伤，氧化还原失衡，进而导致应激性溃疡

[66]。线粒体功能及呼吸功能受损的最常见指标是线粒体呼吸链中复合物的 活性降低, 其中如果复合物 I 的定向呼吸功能受损, 会导致膜电位降低和通透性 转换的敏感度显著增加 $[67][68]$ 。应激也会导致啮齿动物脑中 mtDNAcn 减 少以及 $\mathrm{mtDNA}$ 转录功能变化 $[69$ 。 。其他研究者也报告了心理应激对线粒体基 因转录产生负面影响 [70 ]。有证据表明 mtDNA 的遗传变异可能会影响线粒体 对心理应激反应的易感性 [71]。通过代谢组学领域的研究证据表明，处于应 激状态动物的大脑和血液中线粒体的产生和分泌发生了显著变化, 而不处于应 激状态的对照组动物的线粒体则无显著变化 [72]。此外, 线粒体的蛋白质组 成在应激与非应激条件下会发生显著变化 [73]。

通过大量使用动物和人类为对象的实验研究表明, 不论是急性还是慢性心 
理应激，都会对 mtDNA 功能以及机体产生多种病理性影响。因此，今后尝试使 用线粒体含量及其功能水平作为心理应激反应的指标，不仅能够对心理应激进 行客观、有效的评估，而且能够对多种心身疾病的诊断提供有效参考。

\section{2 线粒体 DNA 拷贝数及其他线粒体功能指标的采集和运} 用

线粒体 DNA 拷贝数是一种间接评估线粒体功能的方法。mtDNAcn 及其他线 粒体功能指标在心理应激暴露时即会发生变化, 其变化往往预示着患上一些心 身疾病的危险性提升，如果将其引人心理应激研究中，将有利于及时监测心理 应激暴露人群的心身健康水平，为后期的危机干预和健康管理提供有效的预警。

关于 mtDNAcn 的测定方法较为简单便捷，适用于大规模人群为样本的研究。 目前对于 mtDNAcn 的检测, 通常采用简单有效的实时定量聚合酶链反应（PCR） 技术进行，所需的样本量较少， $1 \mathrm{pg}$ 的 DNA 样本即可测定 mtDNAcn [ 74]，未 来随着技术的不断进步，在自然灾害或特大人为安全事故的情况下能够进行大 规模的检测。

对于线粒体 DNA 功能而言，线粒体呼吸容量可以作为有效反应线粒体 DNA 功能状态的有效指标。如果要评估线粒体呼吸容量，可以直接在新鲜细胞中检 测其呼吸运动 $[75][76]$ ，或者间接地从冷冻的样品中通过呼吸链复合物的酶 活性来进行检测 [ 77 ]。另外, 线粒体ATP合成也可以直接在新鲜细胞中测量 $[78$ 。

有研究者提出使用粒体健康指数（Index of Mitochondrial Health，MHI）来反 映慢性应激对线粒体健康的影响 [79］。对于反映线粒体功能而言，使用 MHI 效果优于线粒体单个功能测量。因此, 使用数学整合的方法把 mtDNAcn 及其功 能结合起来成为一个客观指标，将有效地提高线粒体作为一种生物标记物对心 理应激反应的评估效果。

探索替代问卷或量表测量进行心理应激暴露水平评估的客观方法，一直以 来都是心理应激领域研究的重点。MtDNAcn 指标相对于其他评估应激的生理指 标（例如头发皮质醇、心率变异性）用途广泛，其不仅能反映慢性心理应激状 况 $[80][81][82]$ ，也能作为急性心理应激的评估指标 $[83]$ [84］。特别 
是在发生重大自然灾害或安全事故时，采用线粒体 DNA 含量及其功能变化的指 标，或许能够更为有效、客观地评估和反应受灾人群的心理应激水平，以便于 后续采取更有针对性的心理援助方案。

\section{5 以线粒体 DNA 作为靶点的心理应激干预策略 探讨}

线粒体动态变化是细胞调节供能, 适应细胞内外环境的重要生理过程 [4]。 线粒体抗氧化能力能够减缓应激带来的影响，国外研究表明，小鼠通过口服线 粒体抗氧化剂能够减缓高焦虑症状的小鼠的焦虑行为 $[85]$ ，甚至可以延长小 鼠的寿命 [86]。这些研究证据间接支持了线粒体功能在应激反应中的作用。 今后仍需要大量的研究来进一步确定, 是否可以通过线粒体靶向干预来预防或 缓解不同应激带来的负性影响，以及这些实验结果是否可以推广、应用于人类 身上。

此外，已知许多生理刺激（例如温度、缺氧、热量摄人等）会对线粒体造 成影响，而耐力运动是对线粒体影响最强且成本最低的刺激。为了适应细胞对 能量需求的变化，线粒体的形态、数目在耐力运动及后续恢复过程中有迅速调 节及表达的变化。具体而言，耐力运动训练增加线粒体含量、线粒体酶的活性 以及肌肉氧化能力 $[87][88][89][90][91]$ 。一项关于啮齿类动物运动 训练的研究表明，增加它们的运动量能够使线粒体 DNA 含量和功能提升 [69］。 既然通过耐力运动可以快速提升线粒体 DNA 含量和功能，也就说明耐力运动对 应激具有缓冲作用可能是通过线粒体功能的变化来介导。值得注意的是，虽然 通过耐力训练能够改善或者减少线粒体功能障碍，但即使是坚持训练的老年人 也在一些指标的改善程度上不如年轻人 [92 ]。这说明了老年人不能通过耐力 训练恢复所有线粒体功能，衰老是导致人体线粒体功能受损重要因素。

因此, 今后的研究应当着重关注和评估通过运动训练导致的线粒体 DNA 含 量和功能变化是否能够有效缓解人类 ( 特别是年轻人) 在心理应激暴露时所带 来的不利影响。在有效控制衰老因素的前提下，围绕提升线粒体健康水平开发 出具有广泛实用性的心理应激干预策略具有重大意义。 


\section{6 研究局限和未来研究展望}

近年来，国外研究者已经开始重视线粒体 DNA 含量及其功能指标作为反映 心理应激的生理指标，且相关研究也逐步展开也已取得一系列支持性证据，但 在以人作为被试的研究过程中也存在一些值得深人探讨的问题，这对国内学者 今后进行心理应激领域的研究定会带来积极的启示。

\section{1 线粒体 DNA 含量功能指标综合使用}

现有以人作为被试的研究主要有五个方面的局限。第一个限制是使用单分 子线粒体标记物，其不直接反映线粒体功能。某些线粒体结果如线粒体 DNA 拷 贝数 $(\mathrm{mtDNAcn})$ 不能直接反映线粒体功能。在缺乏对线粒体功能测量的情况下, mtDNAcn 增加和减少的生理意义尚不清楚。在从全血测量 mtDNAcn 的情况下尤 其如此，全血由具有不同 mtDNA 水平的几种不同的免疫和非免疫细胞类型组成。 动态测量线粒体功能可以与线粒体含量的分子标记同时评估。使用诸如 MHI 这 样综合反映线粒体功能的客观指标，可能比单独测量线粒体某种功能更加敏感 和精确 [79]。有效地提高针对线粒体测量结果的敏感性，将为心理应激源和 情绪状态与线粒体之间的联系提供更为有力的证据。

\section{2 增加线粒体 DNA 检测样本细胞的来源多元化}

血液中免疫细胞组合物与各种应激指标是动态变化的 $[93]$ ［94］，它们 会根据昼夜节律不同发生变化 $[95]$ 。由于每种免疫细胞含有不同数量的线粒 体，这些线粒体表现出不同的功能 [96]。因此，不同白细胞亚群的相对丰度 可以显著改变线粒体测量效果，而在任何细胞类型中线粒体功能却没有任何实 际变化。例如，在血小板的比例发生微小变化时，有助于凝血去核细胞类型的 mtDNA 测量，但如果血小板被污染，外周血单核细胞中 mtDNA 就会发生显著变 化 [97］［98］。未来的研究可通过分离特定的白细胞群来加强测量效果。如 果没办法对不同细胞类型进行分类时，可通过避免血小板污染的方法，这样一 来便可提升线粒体功能数据的可解释性水平。 


\section{3 避免只使用单一组织样本来进行线粒体 DNA 检测}

线粒体存在于所有组织器官类型中, 并且应激诱导的线粒体功能障碍具 有组织特异性。一些社会心理应激源和情绪状态可能优先影响脑部线粒体( 导 致认知衰退或神经障碍）, 而其他可能影响肝脏、心脏内的线粒体（导致代 谢紊乱）。除了使用血液测量之外，其他组织的活组织检查也同样适用于线 粒体检测, 其中包括口腔粘膜细胞、尿液上皮细胞、脂肪和骨骼肌细胞 [ 99 ]。 随着研究技术的发展，采用多种样本来源进行线粒体 DNA 拷贝数和功能的 检测，更能充分验证线粒体 DNA 指标作为心理应激反应指标的客观性和有 效性。

\section{4 实验研究设计要注意纵向追踪和横断设计相结合}

与实验研究相比, 当前的人类研究都对被试的心理应激源经历（例如应 激暴露与否或童年期遭遇应激源）和心理疾病（例如是否存在精神疾病）进行 了测量。这种研究设计很大程度上限制了关于因果关系的探讨, 并且不能排除 很多潜在协变量 (例如运动、睡眠和营养) 的影响。今后研究者可以考虑结合 纵向追踪的实验设计，通过有效控制好潜在的协变量，之后再重复测量线粒体 DNA 含量和功能变化的方法, 来进行关于因果关系的实验探讨。

\section{5 当前研究中的人类被试群体来源不均衡}

与专门针对雄性动物研究正好相反, 人类研究主要关注女性。由于已知线 粒体功能存在性别差异 [95], 在研究设计时需要考虑性别平衡, 以便全面了 解男性和女性在线粒体含量和功能上细节性差异。此外, 到目前为止, 人类研 究主要集中在年轻人和中年人身上, 并且集中于一些特定的群体 (例如, 儿童 期经历创伤者、自杀未遂者、医院护理人员以及孕妇），还没有深人地关注到 不同国家，不同种族、民族间的具体差异。在未来的研究中充分考虑性别、年 龄和群体变量, 这将有助于建立客观、准确和普遍的心理应激反馈指标，为各 类人群和整个生命周期内的心理应激干预提供更具针对性的干预策略。 


\section{6 综合考虑易导致线粒体 DNA 变异的各类因素}

我国地形呈阶梯型分布，其中以青藏高原为主的第一级阶梯平均海拔在 4000 米以上, 研究者应考虑到高原环境因素对线粒体 DNA 的影响。有研究表 明，在高原习服适应过程中，线粒体的形态、功能、序列等方面会发生显著变 化，mtDNAcn 变化与高原疾病易感性有关 $[100]$ [101 ]。另外，青藏高原是 我国现代构造活动和地震活动最强烈的地区，地震灾害连续发生的过程未见终 止 [102]。因此，今后需进一步结合自然灾害和人为安全事故引发的心理应激 情况，使用 mtDNAcn 及其功能水平作为评估指标，提升对高原地区群众的心身 健康状况的有效评估。未来研究也可以尝试考察平原与高原地区人群在心理应 激时 mtDNAcn 及其功能水平是否存在差异，并探讨其中成因与机制。

\section{7 小结}

未来研究者应重点关注心理应激对人体线粒体含量及功能的具体影响，并 开发出针对不同类型线粒体稳健且快捷的评估方法。此外，在人类研究中，不 同性质的应激源（例如，丧亲、失恋、失业等事件）及其所持续的时间长短（例 如，急性或慢性应激事件），引起个体不同程度（例如，轻度、中度或严重） 的情绪反应（例如，焦虑、抑郁），并且这些应激源可以发生在个体生命的不 同阶段（儿童早期、青少年期、成年期早期、中年期或老年期 ）等变量都需认 真考虑和控制。线粒体和线粒体 DNA 对于心理应激反应的严重程度并不是线性 的 [103]，因此区分不同的应激因素以及由此产生的个体化经验同样重要，并 评估可以独立影响线粒体含量和功能的关键协变量。结合这些因素的前瞻性与 纵向性研究设计将加速推进心理应激领域的发展。

综上所述，线粒体 DNA 作为一种新的心理应激反应指标具有重大意义。为 了促进对生物体内导致代谢失调以及不同的疾病过程有更加全面的理解，研究 者应及时关注线粒体 DNA 含量及其功能指标变化的意义。从而进一步探讨把改 善和提升线粒体功能作为靶向目标的心理应激干预策略的可能性，并针对特定 环境因素 (例如我国青藏高原), 优化线粒体 DNA 作为心理应激反应指标的研究, 
最终为心理应激干预方案制定提供新的方向。

\section{参考文献}

[1] 卜翠萍, 奚耕思. 线粒体与认知老化 $[\mathrm{J}]$. 心理科学进展, 2005，13 (3) : $341-347$.

[2] 王铁英，李静，张叶，等.线粒体 DNA 拷贝数：一种新型的职业与环境暴 露生物标志 $[\mathrm{J}$ ]. 环境与职业医学，2017（6）：560-565.

[ 3 ] Meyer J N, Leung M C K, Rooney J P, et al. Mitochondria as a Target of Environmental Toxicants [ J ] . Toxicological Sciences, 2013, 134 ( 1 ) : 1-17. https://doi.org/10.1093/toxsci/kft102

[4] Liesa M, Palacin M, Zorzano A. Mitochondrial Dynamics in Mammalian Health and Disease [ J ] . Physiological Reviews, 2009, 89 ( 3 ) : 799-845. https://doi.org/10.1152/physrev.00030.2008

[5] Manoli I, Alesci S, Blackman M R, et al. Mitochondria as key components of the stress response[ $\mathrm{J}]$. Trends in Endocrinology and Metabolism, 2007, 18( 5 ): 190-198. https://doi.org/10.1016/j.tem.2007.04.004

[6] Morava E, Kozicz T. Mitochondria and the economy of stress ( mal )adaptation[ J ]. Neuroscience \& Biobehavioral Reviews, 2013，37 ( 4 ) : 668-680. https://doi.org/10.1016/j.neubiorev.2013.02.005

[ 7 ] Picard M, Juster R P, Mcewen B S. Mitochondrial allostatic load puts the "gluc" back in glucocorticoids [ J ] . Nature Reviews Endocrinology, 2014, 10 ( 5 ) : 303-310. https://doi.org/10.1038/nrendo.2014.22

[ 8 ] Scheffler I E. Mitochondria make a comeback [ J ] . Advanced Drug Delivery Reviews, 2001, 49 ( 1-2) : 3-26.

https://doi.org/10.1016/S0169-409X(01)00123-5

[9] Gorman G S, Chinnery P F, DiMauro S, et al. Mitochondrial diseases [ J ] . Nature Reviews Disease Primers, 2016 ( 2 ) : 1-22.

https://doi.org/10.1038/nrdp.2016.81 
[ 10 ] Morrow R M, Picard M, Derbeneva O, et al. Mitochondrial energy deficiency leads to hyperproliferation of skeletal muscle mitochondria and enhanced insulin sensitivity [ J ] . Proceedings of the National Academy of Sciences , 2017，114 ( 10 ) : 2705-2710. https://doi.org/10.1073/pnas.1700997114

[ 11 ] Wallace D C. Mitochondrial DNA Variation in Human Radiation and Disease[ J ]. Cell, 2015, 163 ( 1 ) : 33-38. https://doi.org/10.1016/j.cell.2015.08.067

[12 ] Hebert-Chatelain E, Desprez T, Serrat, Rom á n, et al. A cannabinoid link between mitochondria and memory $[\mathrm{J}]$. Nature, 2016, 539 ( 7630$): 555$. https://doi.org/10.1016/j.bbabio.2016.04.251

[13 ] 崔荣荣. 心理社会应激的免疫反应规律与机制 $[\mathrm{J}$ ] . 心理科学进展，2019 ( 27 ) : 1-13. https://doi.org/10.1017/S1551929519000129

[ 14 ] Pearce E L, Poffenberger M C, Chang C H, et al. Fueling Immunity: Insights into Metabolism and Lymphocyte Function [ J ] . Science, 2013, 342 ( 6155 ): 1242454. https://doi.org/10.1126/science.1242454

[15] 丰大利, 周福祥. 线粒体 DNA 突变在宫颈癌中的研究探讨 [ J ] . 肿瘤代 谢与营养电子杂志，2018，5（1）：88-91.

[16 ] 刘启梁. 线粒体 DNA 异常与肿瘤 [ J ] . 生命的化学, 2016（6）：862867.

[ 17 ] 张淑萍, 宋书娟, 李雅轩. 人类线粒体 DNA 突变与癌症 $[\mathrm{J}$ ]. 遗传, 2008( 3 ).

[ 18 ] Valko M, Leibfritz D, Moncol J, et al. Free radicals and antioxidants in normal physiological functions and human disease $[\mathrm{J}]$. Int J Bioxhem Cell Biol, 2007, 39 ( 1 ) : 44-84. https://doi.org/10.1016/j.biocel.2006.07.001

[19] Trifunovic A, Wredenberg A, Falkenberg M, et al. Premature ageing in mice expressing defective mitochondrial DNA polymerase $[\mathrm{J}]$. Nature（London ）, 2004, 429 ( 6990 ) : 417-423. https://doi.org/10.1038/nature02517

[ 20 ] Kujoth, G. C. Mitochondrial DNA Mutations, Oxidative Stress, and Apoptosis in Mammalian Aging [ J ] . Science, 2005, 309 ( 5733 ) : 481-484. https://doi.org/10.1126/science.1112125 
[21 ] Taylor R W , Turnbull D M. Mitochondrial DNA mutations in human disease [ J ]. Nat Rev Genet, 2005. 6 ( 5 ) : 389-402.

https://doi.org/10.1038/nrg1606

[22] Latorre-Pellicer A, Moreno-Loshuertos R, Lechuga-Vieco A V, et al. Mitochondrial and nuclear DNA matching shapes metabolism and healthy ageing [ J ] . Nature, 2016. https://doi.org/10.1038/nature18618

[23] Pacak K, Palkovits M. Stressor Specificity of Central Neuroendocrine Responses: Implications for Stress-Related Disorders [ J ] . Endocrine Reviews, 2001, 22 ( 4 ) : 502-548. https://doi.org/10.1210/er.22.4.502

[24] Lupien S J, Mcewen B S, Gunnar M R, et al. Effects of stress throughout the lifespan on the brain, behaviour and cognition $[\mathrm{J}]$. Nature Reviews Neuroscience, 2009, 10 ( 6 ) : 434-445. https://doi.org/10.1038/nrn2639

[25] Mcewen B S. The ever-changing brain: Cellular and molecular mechanisms for the effects of stressful experiences [ J ] . Developmental Neurobiology, 2012, 72 ( 6 ) : 878-890. https://doi.org/10.1002/dneu.20968

[26] 耿柳娜，王雪，相鹏，等. 慢性压力的生理指标：头发皮质醇 $[\mathrm{J}]$. 心理 科学进展，2015，23 ( 10$)$ ：1799-1807.

[27] Mcewen B S. Chapter 3 Protective and damaging effects of stress mediators: central role of the brain [ J ] . Dialogues Clin Neurosci, 2006, 8 ( 4 ) : 367381.

[28 ] Shalev I, Entringer S, Wadhwa P D, et al. Stress and telomere biology: A lifespan perspective [ J ] . Psychoneuroendocrinology, 2013, 38 (9) : 1835-1842. https://doi.org/10.1016/j.psyneuen.2013.03.010

[29] Miller G E. Psychological stress and the human immune system: A metaanalytic study of 30 years of inquiry[ J ]. Psychological Bulletin, 2004, 130( 4 ): 601-630. https://doi.org/10.1037/0033-2909.130.4.601

[30 ] Irwin M R, Cole S W. Reciprocal regulation of the neural and innate immune systems [ J ] . Nature Reviews Immunology, 2011, 11（9）: 625-632. 
https://doi.org/10.1038/nri3042

[ 31 ] Slavich G M, Cole S W. The Emerging Field of Human Social Genomics [ J ] . Clinical Psychological Science, 2013, 1 ( 3 ) : 331-348.

https://doi.org/10.1177/2167702613478594

[ 32 ] Choi S, Chen M, Cryns V L, et al. A nuclear phosphoinositide kinase complex regulates [ J ] . Nature Cell Biology, 2019: 53.

https://doi.org/10.1038/s41556-019-0297-2

[ 33 ] Cole S W, Nagaraja A S, Lutgendorf S K, et al. Sympathetic nervous system regulation of the tumour microenvironment $[\mathrm{J}]$. Nature Reviews Cancer, 2015, 15 ( 9 ) : 563-572. https://doi.org/10.1038/nrc3978

[ 34 ] Aya Sasaki, Wilfred C. de Vega, Patrick O. McGowan. Biological embedding in mental health: An epigenomic perspective $1[\mathrm{~J}]$. Biochemistry and Cell Biology, 2013, 91 ( 1 ) : 14-21. https://doi.org/10.1139/bcb-2012-0070

[35 ] Marija K, Champagne F A. Early-life experience, epigenetics, and the developing brain $[\mathrm{J}]$. Neuropsychopharmacology Official Publication of the American College of Neuropsychopharmacology, 2016, 55 ( 10 ) : 141-153.

[ 36 ] Brunst K J, Sanchez Guerra M, Gennings C, et al. Maternal lifetime stress and prenatal psychological functioning are associated with decreased placental mitochondrial DNA copy number in the PRISM study [ J ] . American Journal of Epidemiology, 2017. https://doi.org/10.1093/aje/kwx183

[37 ] Morris G, Berk M, Maes M, et al. Socioeconomic Deprivation, Adverse Childhood Experiences and Medical Disorders in Adulthood: Mechanisms and Associations [ J ] . Molecular neurobiology, 2019.

https://doi.org/10.1007/s12035-019-1498-1

[38 ] Menshikova E V, Ritov V B, Fairfull L, et al. Effects of Exercise on Mitochondrial Content and Function in Aging Human Skeletal Muscle [ J ] . The Journals of Gerontology Series A: Biological Sciences and Medical Sciences, 2006, 61 ( 6 ) : 534-540. https://doi.org/10.1093/gerona/61.6.534 
[ 39 ] Lanza I R, Nair K S. Regulation of skeletal muscle mitochondrial function: genes to proteins $[\mathrm{J}]$. Acta Physiologica, 2010, 199 ( 4 ) : 529-547. https://doi.org/10.1111/j.1748-1716.2010.02124.x

[40] 刘健康, 王学敏.线粒体医学与健康 [M]. 北京：科学出版社, 2012.

[41] 张素素, 孙嘉. 线粒体 DNA 拷贝数的研究新进展 [ J ]. 医学综述, 2014( 24 ): 4417-4419.

[42 ] 陈念, 赵树进，韩丽萍，等. 线粒体 DNA 拷贝数变异机制研究进展 $[\mathrm{J}]$. 医学研究生学报，2008，21（7）：771-774.

[ 43 ] Montier L L C, Deng J J, Bai Y. Number matters: control of mammalian mitochondrial DNA copy number $[\mathrm{J}]$. Journal of Genetics and Genomics, 2009, 36 ( 3 ) : 125-131. https://doi.org/10.1016/S1673-8527(08)60099-5

[44] Giles R E, Blanc H, Cann H M, et al. Maternal inheritance of human mitochondrial DNA $[\mathrm{J}]$. Proceedings of the National Academy of Sciences of the United States of America, 1980, 77 ( 11 ) : 6715-6719. https://doi.org/10.1073/pnas.77.11.6715

[45] Kenney M C, Chwa M, Atilano S R, et al. Molecular and bioenergetic differences between cells with African versus European inherited mitochondrial DNA haplogroups: Implications for population susceptibility to diseases $[\mathrm{J}]$. Biochimica et Biophysica Acta (BBA ) - Molecular Basis of Disease, 2014, 1842 ( 2 ) : 208-219. https://doi.org/10.1016/j.bbadis.2013.10.016

[ 46 ] Chan S R W L, Blackburn E H. Telomeres and telomerase [ J ] . Philosophical Transactions of the Royal Society of London, 2004, 359 ( 1441 ) : 109-121. https://doi.org/10.1098/rstb.2003.1370

[47 ] Yakes F M, Van Houten B. Mitochondrial DNA damage is more extensive and persists longer than nuclear DNA damage in human cells following oxidative stress [ J ] . Proceedings of the National Academy of Sciences, 1997, 94 ( 2 ): 514-519. https://doi.org/10.1073/pnas.94.2.514

[48] 赵云罡, 徐建兴. 线粒体, 活性氧和细胞凋亡 $[\mathrm{J}]$. 生物化学与生物物理 
进展，2001，28（2）：168-171.

[49] Mitchell P. Chemiosmotic hypothesis of oxidative phosphorylation [ J ] . Nature, 1967, 213 ( 5072 ) : 137-139. https://doi.org/10.1038/213137a0

[50 ] 李阳, 郭瑶, 刘中英, 等. 应激引发线粒体功能障碍的研究进展 $[\mathrm{J}]$. 黑 龙江畜牧兽医，2018：57-59.

[51] Mcmanus M J, Murphy M P, Franklin J L. The Mitochondria-Targeted Antioxidant MitoQ Prevents Loss of Spatial Memory Retention and Early Neuropathology in a Transgenic Mouse Model of Alzheimer"s Disease [ J ] . Journal of Neuroscience, 2011, 31 (44) : 15703-15715. https://doi.org/10.1523/JNEUROSCI.0552-11.2011

[ 52 ] Mcewen B S. Perturbing the Organism: The Biology of Stressful Experience [ J ] . JAMA The Journal of the American Medical Association, 1993, 269 ( 10 ) : 1315. https://doi.org/10.1001/jama.1993.03500100115046

[53 ] Gimsa U, Kanitz E, Otten W, et al. Behavior and Stress Reactivity in Mouse Strains with Mitochondrial DNA Variations [ J ] . Annals of the New York Academy of Sciences, 2009, 1153 ( 1 ) : 131-138. https://doi.org/10.1111/j.1749-6632.2008.03960.x

[54] Picard M, Mcmanus M J, Gray J D, et al. Mitochondrial functions modulate neuroendocrine, metabolic, inflammatory, and transcriptional responses to acute psychological stress $[\mathrm{J}]$. Proceedings of the National Academy of Sciences, 2015, 112 ( 48 ) : 6614-6623.

https://doi.org/10.1073/pnas.1515733112

[ 55 ] Meimaridou E, Kowalczyk J, Guasti L, et al. Mutations in NNT encoding nicotinamide nucleotide transhydrogenase cause familial glucocorticoid deficiency $[\mathrm{J}]$. Nature Genetics, 2012, 44 ( 7 ) : 740-742. https://doi.org/10.1038/ng.2299

[56] Severity of cardiomyopathy associated with adenine nucleotide translocator-1 deficiency correlates with mtDNA haplogroup $[\mathrm{J}]$. Proceedings of the National 
Academy of Sciences, 2013, 110 ( 9 ) : 3453-3458.

https://doi.org/10.1073/pnas.1300690110

[57] Demonacos C, Djordjevic-Markovic R, Tsawdaroglou N, et al. The mitochondrion as a primary site of action of glucocorticoids : the interaction of the glucocorticoid receptor with mitochondrial DNA sequences showing partial similarity to the nuclear glucocorticoid responsive elements $[\mathrm{J}]$. Journal of Steroid Biochemistry and Molecular Biology, 1995, 55 ( 1 ) : 43-55. https://doi.org/10.1016/0960-0760(95)00159-W

[ 58 ] Michael L F, Wu Z, Cheatham R B, et al. Restoration of insulin-sensitive glucose transporter ( GLUT4 ) gene expression in muscle cells by the transcriptional coactivator PGC-1 [ J ] . Proceedings of the National Academy of Sciences, 2001, 98 ( 7 ) : 3820-3825.

https://doi.org/10.1073/pnas.061035098

[59 ] Gullicksen P S, Feng Y, Wu Z, et al. Transducer of regulated CREB-binding proteins ( TORCs ) induce PGC-1alpha transcription and mitochondrial biogenesis in muscle cells [ J ] . Proc Natl Acad Sci U S A, 2006, 103 ( 39 ) : 14379-14384. https://doi.org/10.1073/pnas.0606714103

[60 ] Taivassalo, T. The spectrum of exercise tolerance in mitochondrial myopathies: a study of 40 patients [ J ] . Brain, 2003, 126 ( 2 ) : 413-423. https://doi.org/10.1093/brain/awg028

[61] Bates M G D, Newman J H, Jakovljevic D G, et al. Defining cardiac adaptations and safety of endurance training in patients with $\mathrm{m}$. 3243A $>\mathrm{G}-$ related mitochondrial disease $[\mathrm{J}]$. International Journal of Cardiology, 2013, 168 ( 4 ) : 3599-3608. https://doi.org/10.1016/j.ijcard.2013.05.062

[62 ] Jeppesen T D, Orngreen M C, Van Hall G, et al. Lactate metabolism during exercise in patients with mitochondrial myopathy $[\mathrm{J}]$. Neuromuscular Disorders, 2013, 23 ( 8 ) : 629-636.

https://doi.org/10.1016/j.nmd.2013.05.007 
[63 ] Singh A, Petrides J S, Gold P W, et al. Differential Hypothalamic-PituitaryAdrenal Axis Reactivity to Psychological and Physical Stress [ J ] . The Journal of Clinical Endocrinology \& Metabolism, 1999, 84 ( 6 ) : 1944-1948. https://doi.org/10.1210/jc.84.6.1944

[64 ] Desantis A S, Adam E K, Hawkley L C, et al. Racial and Ethnic Differences in Diurnal Cortisol Rhythms: Are They Consistent Over Time? [ J ] . Psychosomatic Medicine, 2014, 77 ( 1 ) : 6-15.

https://doi.org/10.1097/PSY.0000000000000131

[65 ] Picard M, Mcewen B S. Psychological Stress and Mitochondria [J ] . Psychosomatic Medicine, 2018, 80 ( 2 ) : 126-140.

\section{https://doi.org/10.1097/PSY.0000000000000544}

[66 ] De R, Mazumder S, Sarkar S, et al. Acute mental stress induces mitochondrial bioenergetic crisis and hyper-fission along with aberrant mitophagy in the gut mucosa in rodent model of stress-related mucosal disease $[\mathrm{J}]$. Free Radical Biology and Medicine, 2017.

[67 ] Rezin G T, Cardoso M R, Goncalves C L, et al. Inhibition of mitochondrial respiratory chain in brain of rats subjected to an experimental model of depression [ J ] . Neurochemistry International, 2008, 53 ( 6 ) : 395-400. https://doi.org/10.1016/j.neuint.2008.09.012

[68 ] Madrigal J L, Olivenza R, Moro M A, et al. Glutathione Depletion, Lipid Peroxidation and Mitochondrial Dysfunction Are Induced by Chronic Stress in Rat Brain [ J ] . Neuropsychopharmacology, 2001, 24 ( 4 ) : 420-429. https://doi.org/10.1016/S0893-133X(00)00208-6

[69] Liu W, Zhou C. Corticosterone reduces brain mitochondrial function and expression of mitofusin, BDNF in depression-like rodents regardless of exercise preconditioning $[\mathrm{J}]$. Psychoneuroendocrinology, 2012, 37 ( 7 ) : 1057-1070. https://doi.org/10.1016/j.psyneuen.2011.12.003

[ 70 ] Hunter R G, Seligsohn, Ma'ayan, Rubin T G, et al. Stress and corticosteroids 
regulate rat hippocampal mitochondrial DNA gene expression via the glucocorticoid receptor $[\mathrm{J}]$. Proceedings of the National Academy of Sciences, 2016, $113(32)$ : 9099-9104.

https://doi.org/10.1073/pnas.1602185113

[ 71 ] Garc í a-Fern á ndez, Mar í a, Castilla-Ortega E, Pedraza C, et al. Chronic Immobilization in the ma\r, lpar $1 \backslash r$, Knockout Mice Increases Oxidative Stress in the Hippocampus[ J ]. International Journal of Neuroscience, 2012, 122( 10 ). 583-589. https://doi.org/10.3109/00207454.2012.693998

[72 ] Johnson C H, Ivanisevic J, Siuzdak G. Metabolomics: beyond biomarkers and towards mechanisms[ J ]. Nature Reviews Molecular Cell Biology, 2016,17( 7 ): 451-459. https://doi.org/10.1038/nrm.2016.25

[73 ] Filiou M D, Zhang Y, Teplytska L, et al. Proteomics and Metabolomics Analysis of a Trait Anxiety Mouse Model Reveals Divergent Mitochondrial Pathways [J ] . Biological psychiatry, 2011, 70 ( 11 ) : 1074-1082. https://doi.org/10.1016/j.biopsych.2011.06.009

[74] Malik A N, Shahni R, Rodriguez-De-Ledesma A, et al. Mitochondrial DNA as a non-invasive biomarker: Accurate quantification using real time quantitative PCR without co-amplification of pseudogenes and dilution bias $[\mathrm{J}]$. Biochemical \& Biophysical Research Communications, 2011, 412 ( 1 ) : 1-7. https://doi.org/10.1016/j.bbrc.2011.06.067

[75] Kuznetsov A V, Veksler V, Gellerich F N, et al. Analysis of mitochondrial function in situ in permeabilized muscle fibers, tissues and cells $[\mathrm{J}]$. Nature Protocols, 2008, 3 ( 6 ) : 965-976. https://doi.org/10.1038/nprot.2008.61

[76 ] Lanza I R, Nair K S. Mitochondrial metabolic function assessed in vivo and in vitro [ J ] . Current Opinion in Clinical Nutrition and Metabolic Care, 2010, 13 ( 5 ) : 511-517. https://doi.org/10.1097/MCO.0b013e32833cc93d

[77 ] Medja F, Allouche S, Frachon P, et al. Development and implementation of standardized respiratory chain spectrophotometric assays for clinical diagnosis. 
[ J ] . Mitochondrion, 2009, 9 ( 5 ) : 331-339.

https://doi.org/10.1016/j.mito.2009.05.001

[78 ] Booth N E, Myhill S, Mclarenhoward J. Mitochondrial dysfunction and the pathophysiology of Myalgic Encephalomyelitis/Chronic Fatigue Syndrome（ ME/ CFS ) . [ J ] . International Journal of Clinical \& Experimental Medicine, 2012, 5 ( 3 ) : 208-220.

[79] Picard M, Prather A A, Puterman E, et al. A Mitochondrial Health Index Sensitive to Mood and Caregiving Stress[ J ]. Biological Psychiatry, 2018, 84( 1 ). 9-17. https://doi.org/10.1016/j.biopsych.2018.01.012

[ 80 ] Tyrka A R, Parade S H, Price L H, et al. Alterations of Mitochondrial DNA Copy Number and Telomere Length with Early Adversity and Psychopathology. [ J ] . Biological Psychiatry, 2016, 79 ( 2 ) : 78-86. https://doi.org/10.1016/j.biopsych.2014.12.025

[81] Boeck C, Koenig A M, Schury K, et al. Inflammation in adult women with a history of child maltreatment: The involvement of mitochondrial alterations and oxidative stress [ J ] . Mitochondrion, 2016, 30: 197-207. https://doi.org/10.1016/j.mito.2016.08.006

[ 82 ] Cai N, Chang S, Li Y, et al. Molecular Signatures of Major Depression [ J ] . Current biology: CB, 2015, 25 ( 9 ) : 1146-1156.

https://doi.org/10.1016/j.cub.2015.03.008

[83] Batandier C, Poulet L, Hininger I, et al. Acute stress delays brain mitochondrial permeability transition pore opening $[\mathrm{J}]$. Journal of Neurochemistry, 2014, 131 ( 3 ) : 314-322.

https://doi.org/10.1111/jnc.12811

[ 84 ] Andric S A, Kojic Z, Bjelic M M, et al. The opposite roles of glucocorticoid and alpha1-adrenergic receptors in stress triggered apoptosis of rat Leydig cells [ J ] . American Journal of Physiology Endocrinology \& Metabolism, 2013, 304 ( 1 ) : 51-59. https://doi.org/10.1152/ajpendo.00443.2012 
[ 85 ] Nussbaumer M, Asara J M, Teplytska L, et al. Selective Mitochondrial Targeting Exerts Anxiolytic Effects In Vivo [ J ] . Neuropsychopharmacology, 2016, 41 ( 7 ) : 1751-1758. https://doi.org/10.1038/npp.2015.341

[ 86 ] Schriner, S. E. Extension of Murine Life Span by Overexpression of Catalase Targeted to Mitochondria [ J ] . Science, 2005, 308 ( 5730 ) : 1909-1911. https://doi.org/10.1126/science.1106653

[87 ] Chow L S, Greenlund L J, Asmann Y W, et al. Impact of endurance training on murine spontaneous activity, muscle mitochondrial DNA abundance, gene transcripts, and function [ J ] . Journal of Applied Physiology, 2007, 102 ( 3 ): 1078-1089. https://doi.org/10.1152/japplphysiol.00791.2006

[ 88 ] Short K R, Vittone J L, Bigelow M L, et al. Impact of Aerobic Exercise Training on Age-Related Changes in Insulin Sensitivity and Muscle Oxidative Capacity [ J ] . Diabetes, 2003, 52 ( 8 ) : 1888-1896.

https://doi.org/10.2337/diabetes.52.8.1888

[ 89 ] Kent-Braun J A, Mccully K K, Chance B. Metabolic effects of training in humans: a 31P-MRS study [ J ]. Journal of Applied Physiology, 1990, 69 ( 3 ): 1165-1170. https://doi.org/10.1152/jappl.1990.69.3.1165

[90 ] Davies K J, Packer L, Brooks G A. Biochemical adaptation of mitochondria, muscle, and whole-animal respiration to endurance training. $[\mathrm{J}]$. Archives of Biochemistry \& Biophysics, 1981, 209 ( 2 ) : 539-554.

https://doi.org/10.1016/0003-9861(81)90312-X

[91 ] Holloszy J O. Biochemical Adaptations in Muscle [ J ] . Journal of Biological Chemistry, 1967, $242(9): 2278-2282$.

[92] Lanza I R, Short D K, Short K R, et al. Endurance Exercise as a Countermeasure for Aging [ J ] . Diabetes, 2008, 57 ( 11 ) : 2933-2942. https://doi.org/10.2337/db08-0349

[93 ] Dhabhar F S, Mcewen B S. Acute Stress Enhances while Chronic Stress Suppresses Cell-Mediated Immunityin Vivo: A Potential Role for Leukocyte 
Trafficking [ J ] . Brain Behavior \& Immunity, 1997, 11 ( 4 ) : 286-306. https://doi.org/10.1006/brbi.1997.0508

[94 ] Dhabhar F S, Malarkey W B, Neri E, et al. Stress-induced redistribution of immune cells-From barracks to boulevards to battlefields: A tale of three hormones - Curt Richter Award Winner [ J ] . Psychoneuroendocrinology, 2012, 37 ( 9 ) : 1345-1368. https://doi.org/10.1016/j.psyneuen.2012.05.008

[95 ] Born J ,, Lange T ,, Hansen K ,, et al. Effects of sleep and circadian rhythm on human circulating immune cells[ J ]. Journal of Immunology, 1997, 158( 9 ): 4454-4464.

[96] Chacko B K, Kramer P A, Ravi S, et al. Methods for defining distinct bioenergetic profiles in platelets, lymphocytes, monocytes, and neutrophils, and the oxidative burst from human blood $[\mathrm{J}]$. Laboratory Investigation, 2013, 93 ( 6 ) : 690-700. https://doi.org/10.1038/labinvest.2013.53

[97 ] Urata M, Koga-Wada Y, Kayamori Y, et al. Platelet contamination causes large variation as well as overestimation of mitochondrial DNA content of peripheral blood mononuclear cells $[\mathrm{J}]$. Annals of Clinical Biochemistry, 2008, 45 ( 5 ) : 513-514. https://doi.org/10.1258/acb.2008.008008

[ 98 ] Hurtadoroca Y, Ledesma M, Gonzalezlazaro M, et al. Adjusting MtDNA Quantification in Whole Blood for Peripheral Blood Platelet and Leukocyte Counts [ J ]. Plos One, 2016, 11 ( 10$)$. https://doi.org/10.1371/journal.pone.0163770

[99] Janssen A J M. Measurement of the Energy-Generating Capacity of Human Muscle Mitochondria: Diagnostic Procedure and Application to Human Pathology [ J ] . Clinical Chemistry, 2006, 52 ( 5 ) : 860-871. https://doi.org/10.1373/clinchem.2005.062414

[100 ] 罗勇军，刘福玉，陈丽，等. 血细胞线粒体 DNA 拷贝数变化与高原红细 胞增多症易感性的关联研究 [ J ] . 国际检验医学杂志, 2011，32（11）: $1176-1177$. 
[101］罗勇军，高钰琪. 线粒体基因组变异与高原环境适应相关性的研究进展 [J ] . 国际遗传学杂志，2011，34（1）：27-30.

[102］邓起东，程绍平，马冀，等.青藏高原地震活动特征及当前地震活动形 势 $[\mathrm{J}$ ].期刊，2015，57（7）：2025-2042.

[ 103 ] Mitochondrial DNA copy number is reduced in male combat veterans with PTSD [ J ] . Progress in Neuro-Psychopharmacology and Biological Psychiatry, 2016, 64: 10-17. https://doi.org/10.1016/j.pnpbp.2015.06.012 